\title{
Ergogenic Capacity of a 7-Chinese Traditional Medicine Extract in Aged Mice
}

\author{
Jian-Rong Zhou' ${ }^{1}$, Mohamed Aly M. Morsy ${ }^{2}$, Kiyoshi Kunika ${ }^{3}$, \\ Kazumi Yokomizo ${ }^{1}$, Takeshi Miyata ${ }^{1}$ \\ ${ }^{1}$ Department of Presymptomatic Medical Pharmacology, Faculty of Pharmaceutical Sciences, Sojo University, Kumamoto, Japan \\ ${ }^{2}$ Department of Pharmacology, Faculty of Medicine, Minia University, Minya, Egypt \\ ${ }^{3}$ Division of Science and Medicine, Institute of International Kampo Co. Ltd., Nihonmatsu, Japan \\ Email: zhoujr@ph.sojo-u.ac.jp
}

Received October 6, 2012; revised November 9, 2012; accepted November 17, 2012

\begin{abstract}
The ergogenic properties of a 7-Chinese traditional medicine water extract (Ligustrum lucidum ait, LLA), which is composed of the essences of Lycii fructus, Crataegi fructus, Phyllanthi fructus, Chrysanthemi flos, Coicis semen, Ganoderma lucidum, and Zizyphi fructus, were studied using aged mice. Mice were chronically (one month) administered LLA $(0.1 \%$ and $1 \%)$ in the drinking water. Mice pre-treated with LLA showed a good appetite; however, they exhibited a lower rate of body weight increase compared to control mice. In mice subjected to the rotarod test, $1 \%$ LLA treatment provided effective adaption to fatigue and significantly increased the duration of mice on the rotarod. In locomotor activity test, $1 \%$ LLA potentiated mice mobility and significantly increased rearing behavior. In the antioxidant experiment, 1\% LLA treatment significantly increased superoxide dismutase activity in the spleen and liver glutathione levels. These findings suggest that LLA may be utilized as an antifatigue agent, which may function through its antioxidant activity.
\end{abstract}

Keywords: Antifatigue; Antioxidant; Chinese Traditional Medicine

\section{Introduction}

A 7-Chinese traditional medicine water extract (Ligustrum lucidum ait, LLA) has been used for more than thirty years in China and Japan, and is composed of the essences of Lycii fructus from Lycium chinense Miller, Crataegi fructus from Crataegus cuneata Siebold et Zuccarini, Phyllanthi fructus from Phyllanthus emblica L., Chrysanthemi flos from Chrysanthemum morifolium, Zizyphi fructus from Zizyphus jujuba Miller var. inermis Rehd, Ganoderma lucidum, and Coicis semen from Coix lacryma-jobi L. var. mayuen Stapf. In an in vitro experiment with aged mice, it was demonstrated that at a lower concentration LLA could act as a co-stimulator with mitogens, whereas at a higher concentration it was able to act as an immunosuppressive agent [1]. In a clinical study, Chien et al. reported that after administration of LLA, IL-2 levels increased and decreased levels of lymphocyte transformation returned to normal levels in aged volunteers. Follow-up medical examinations showed that inspiration $(100 \%)$, good appetite $(95 \%)$, sexual $(35 \%)$ and sound sleep $(95 \%)$ were the general clinical manifestations [1]. We are interested in the improved general clinical manifestations following LLA treatment; however, it is still unknown whether the underlying pharma- cological mechanism involves immunomodulation and/or other pathways.

The main nutrient compositions of LLA are listed in Table 1, and the most important thing is that LLA contains a variety of components with antioxidant activities, such as Lycium, Crataegus, Emblica, and Ganoderma, and their antioxidant activities have been well described. It has been reported that polysaccharides from Lycium chinense, a component of LLA, improved superoxide dismutase (SOD) activity in damaged rat testes, and its antioxidant activities were comparable to the normal antioxidant, vitamin $\mathrm{C}[2,3]$. An increasing number of reports have also suggested that Ganoderma, which has multiple health benefits for a broad range of conditions (from arthritis to cancers), has antioxidant properties as a free radical scavenger $[4,5]$. Furthermore, it was found that Emblica, another component of LLA, had a higher content of vitamin C, accounting for $45 \%-70 \%$ of the antioxidant activity [6]. Dicaffeoylquinic acids from Chrysanthemi Flos and six phenolic compounds from Coicis Semen showed potent DPPH radical scavenging activity $[7,8]$. These may contribute greatly to the antioxidant property of LLA.

Although LLA has a significant folk history in China 
Table 1. Mean values of the nutrient composition (amino acid, mineral, vitamine and sugar content) for $100 \mathrm{~g}$ LLA extract.

\begin{tabular}{|c|c|c|c|}
\hline Amino acid & Mean $(\mathrm{mg} / \mathbf{1 0 0} \mathrm{g})$ & Mineral & Mean (/100 g) \\
\hline Arginine & 52 & Sodium (Na) & $113.00 \mathrm{mg}$ \\
\hline Lysine & 9 & Phosphorous (P) & $24.60 \mathrm{mg}$ \\
\hline Histidine & 11 & Iron $(\mathrm{Fe})$ & $0.84 \mathrm{mg}$ \\
\hline Phenylanine & 6 & Calcium (Ca) & $13.50 \mathrm{mg}$ \\
\hline Tyrosine & 7 & Potassium (K) & $295.00 \mathrm{mg}$ \\
\hline Leucine & 20 & Magnesium (Mg) & $16.10 \mathrm{mg}$ \\
\hline Isoleucine & 8 & Chlonium $(\mathrm{Cl})$ & $85.00 \mathrm{mg}$ \\
\hline Methionine & 2 & Copper $(\mathrm{Cu})$ & $48.00 \mu \mathrm{g}$ \\
\hline Valine & 16 & Zinc (Zn) & $231.00 \mu \mathrm{g}$ \\
\hline Alanine & 94 & Sulfur (S) & $0.01 \mathrm{~g}$ \\
\hline Glycine & 12 & & \\
\hline Proline & 178 & & \\
\hline Glutamic acid & 156 & Vitamine & Mean (/100 g) \\
\hline Serine & 66 & Vitamine B1 & $0.05 \mathrm{mg}$ \\
\hline Threonine & 31 & Vitamine B2 & $0.07 \mathrm{mg}$ \\
\hline Aspartic acid & 269 & Vitamine B6 & $55.00 \mu \mathrm{g}$ \\
\hline Tryptophan & 6 & Folic acid & $11.00 \mu \mathrm{g}$ \\
\hline \multirow[t]{3}{*}{ Cystine } & 11 & Patoten acid & $0.16 \mathrm{mg}$ \\
\hline & & Biotin & $4.30 \mu \mathrm{g}$ \\
\hline & Mean (g/100 g) & Niacin & $0.96 \mathrm{mg}$ \\
\hline Sugar content & 10.80 & & \\
\hline
\end{tabular}

and Japan, further scientific investigation of LLA-induced effects is required, as it is important to provide an evidence-based supplement. Therefore, we investigated the antifatigue effect of LLA in aged mice using openfield and rotarod tests, and assessed whether its mechanism of action is related to its antioxidant activity.

\section{Materials and Methods}

\subsection{Animals}

Male ddY mice (SPF grade), 60 weeks old, were obtained from Kyudo Co., Ltd. (Fukuoka, Japan). Mice were housed under the following controlled conditions: temperature $\left(24^{\circ} \mathrm{C} \pm 2^{\circ} \mathrm{C}\right)$, humidity $(50 \% \pm 10 \%)$ and a 12-hour light/dark cycle (7:00 a.m. to 7:00 p.m.). Food and water were available ad libitum. There was a oneweek adaptation period, after which the mice were administered either water or $0.1 \%$ or $1 \%$ LLA. All experiments were conducted in strict accordance with the
Guidelines of the Japanese Pharmacological Society for the Care and Use of Laboratory Animals.

\subsection{Materials}

LLA was prepared and provided by the International Kampo Institute (Nihonmatsu, Japan). SOD Assay KitWST and total GSH Quantification Kits were purchased from Dojindo (Kumamoto, Japan). Other agents were obtained as follows: bovine erythrocyte SOD (Sigma, St. Louis, MO, USA); pentobarbital sodium (Dainippon Sumitomo Pharma, Osaka, Japan); sucrose (Nacalai Tesque, Kyoto, Japan). LLA $(0.1 \%$ and $1 \%)$ were prepared in water.

\subsection{Assay of Antifatigue Endurance Activity Using Rotarod Test}

Either LLA $(0.1 \%$ or $1 \%)$ or water (control group) was administered from water pots for one month. First, the mice were placed on a rotarod (Ugo Basile, Comerio VA, Italy) to induce fatigue for $30 \mathrm{~min}$ at $16 \mathrm{rpm}$. Fatigued mice were again placed on the rotarod $(40 \mathrm{rpm})$ and the riding time (the time before the mice fell off the rod) was determined.

\subsection{Assay of Spontaneous Locomotor Activity Using the Open-Field Test}

The locomotor behavior of mice was measured as previously reported [9]. Briefly, the mice were put into an acrylic cage $(30 \times 36 \times 17 \mathrm{~cm}) 30 \mathrm{~min}$ before the locomotion determination. Lines were drawn on the bottom of the cage, dividing it into nine rectangles. Locomotor activity was evaluated as the ambulation behavior (the number of times a mouse crossed a section) and the rearing behavior (rearing number) during the 5-minute observation period.

\subsection{Assay of Antioxidant Activity Using SOD and GSH Kits}

After one-month LLA administration, mice were sacrificed by decapitation, and blood and tissues were collected. For SOD determination, tissue samples were homogenized in sucrose buffer $(0.25 \mathrm{M}$ sucrose, $10 \mathrm{mM}$ Tris, $1 \mathrm{mM}$ EDTA, $\mathrm{pH}$ 7.4). The homogenates were centrifuged at $10,000 \times \mathrm{g}$ for $15 \mathrm{~min}$ at $4^{\circ} \mathrm{C}$ and supernatants were collected. Bovine erythrocyte SOD was used as the standard. For total GSH determination, serum was mixed with $5 \% 5$-sulfosalicylic acid solution. The mixture was centrifuged at $8000 \times \mathrm{g}$ for $10 \mathrm{~min}$ at $4^{\circ} \mathrm{C}$ and the supernatant was collected. Tissue samples were homogenized in 5\% 5-sulfosalicylic acid solution. The homogenates were centrifuged at $8000 \times \mathrm{g}$ for $10 \mathrm{~min}$ at $4^{\circ} \mathrm{C}$, and the supernatants were collected. SOD and total 
GSH activities were determined in triplicate with colorimetric assays, following the manufacturer's instructions. For tissues, SOD and total GSH were expressed as units per mg protein and $\mu \mathrm{M}$ per mg protein relative to the standards, respectively. Protein concentration was measured using the Bradford method.

\subsection{Statistical Analysis}

Each value represents the mean \pm SEM for 5 - 6 mice. The data were statistically evaluated using student's ttest. Probability $(p)$ values less than 0.05 were considered to be statistically significant.

\section{Results}

\subsection{Changes in Food Intake and Weight}

The LLA group consumed more food than the control group (Figure 1(a)), while the rate of body weight increase in the LLA group showed a decreasing tendency after 15 days of administration (Figure 1(b)).

\subsection{Extension of Rotarod Riding Time}

We studied the antifatigue effect of LLA on aged mice using the rotarod test. As shown in Figure 2, the rotarod riding time in the LLA group was increased $2.8 \pm 1.6$ and $6.7 \pm 1.4$ times on the 20th and 30th day of administration, respectively. The time measured on the 30th day was significantly longer $(p<0.05)$ compared to the control mice.

\subsection{Excitory Effects on Spontaneous Locomotor Behavior}

As shown in Figure 3, ambulation and rearing behaviors in the LLA group for 5 min were $45.4 \pm 11.2$ and $25.5 \pm$ 5.5 counts, respectively, and those of the control group were $20.4 \pm 6.6$ and $7.5 \pm 2.3$ counts, respectively. LLA induced an increase in ambulation and rearing behaviors in mice, and the increase in rearing behavior was significant $(p<0.05)$.

\subsection{Antioxidant Activity}

To determine whether the supplementation of LLA demonstrates ergogenic performance by improving the oxidant status in mice, we assessed the SOD and total GSH activities of LLA in aged mice serum and tissues.

As shown in Figure 4(a), the spleen SOD level was elevated significantly by LLA; level was $1.6 \pm 0.1$ and $2.4 \pm 0.2$ units $/ \mathrm{mg}$ protein in the control and LLA groups, respectively. The testes SOD level in LLA mice showed an increasing tendency; the level was $6.0 \pm 1.0$ and $10.8 \pm$ 2.9 units/mg protein in the control and LLA groups, respectively. On the other hand, there were no significant

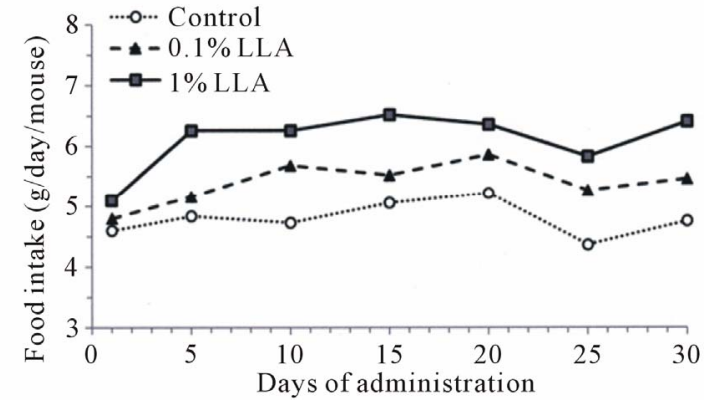

(a)

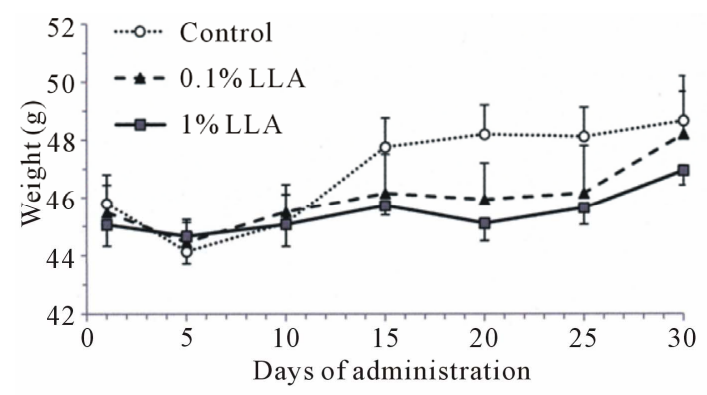

(b)

Figure 1. Effects of LLA supplementation (versus the control group receiving water) on food intake and weight in aged mice. LLA (1\%) and water (control group) were administered from water pots for one month. Food and water were available ad libitum $(\mathrm{n}=5)$.

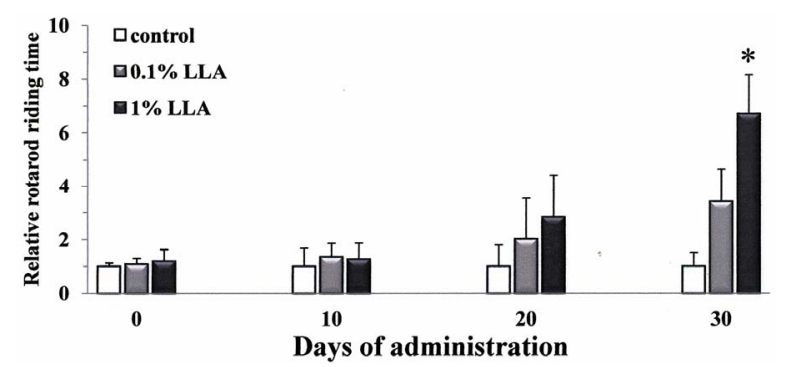

Figure 2. Chronic intake of LLA extends rotarod riding time in aged mice. Data are means $\pm \operatorname{SEM}(n=4-5) .{ }^{*} p<$ 0.05, significantly different from the control.

differences in brain, liver and kidney SOD levels between the control and LLA groups. The serum SOD level was $25.7 \pm 11.7$ and $27.5 \pm 4.9$ units $/ \mathrm{ml}$ in the control and LLA groups, respectively (data not shown).

For total GSH activity, LLA significantly increased liver GSH levels (Figure 4(b)) in the control $(0.1 \pm 0.02$ $\mu \mathrm{M} / \mathrm{mg}$ protein $)$ and LLA $(0.2 \pm 0.02 \mu \mathrm{M} / \mathrm{mg}$ protein $)$ groups. However, there were no significant differences in the total GSH levels of serum and other tissues between the two groups (data not shown).

\section{Discussion}

In the present study, chronic administration of LLA demonstrated an ergogenic property, possibly mediated 


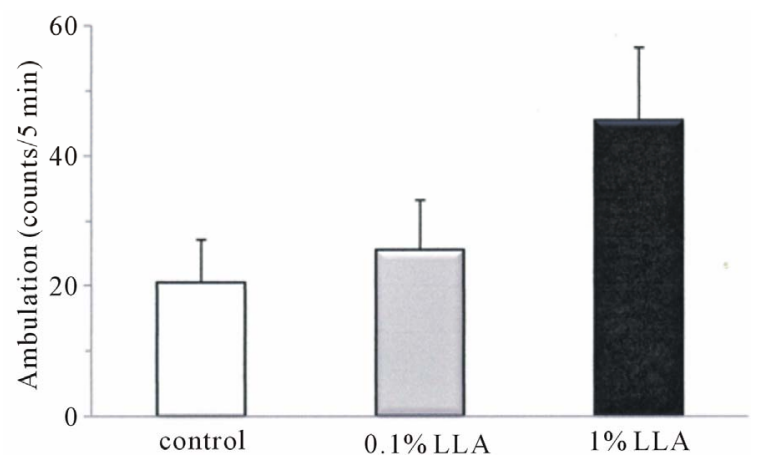

(a)

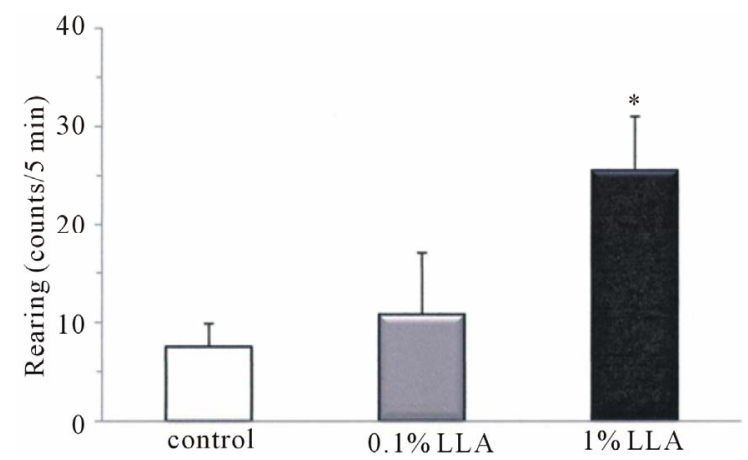

(b)

Figure 3. Effects of LLA treatment on spontaneous locomotor activity. Data are means \pm SEM $(n=4 \sim 5) .{ }^{*} p<0.05$, significantly different from the control.

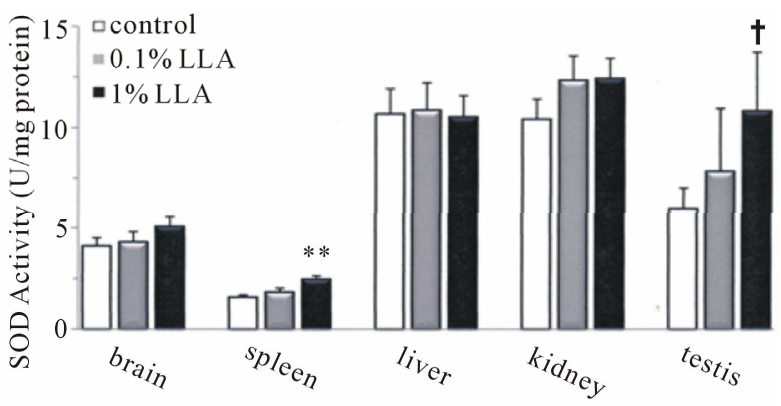

(a)

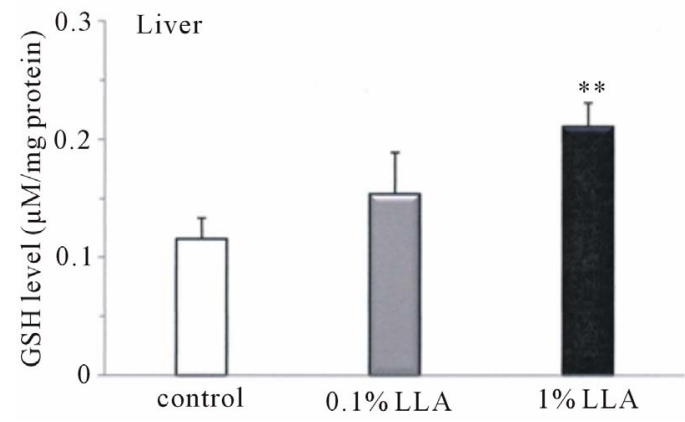

(b)

Figure 4. Antioxidant activity in tissues from aged mice after chronic intake of LLA. Data are means \pm SEM $(n=4 \sim$ 5). ${ }^{* *} p<0.01$, significantly different from the control. ${ }^{\dagger} p<$ 0.1 , tendency differing from control. by functioning in an antioxidative system.

In the in vivo experiment, aged mice had a good appetite following LLA treatment (Figure 1). Preliminary clinical studies also suggest LLA supplementation had appetizing effects. Despite the increased food intake, the rate of increase in body weight of LLA mice was not as high as that of control mice. This may be important effect for humans, since the trend of increasing rates of obesity appears to be unwavering, and the most effective, currently available, treatment for severe obesity is obesity surgery. It has been reported that Crataegus fructus, one of the components in LLA, markedly reduces food intake, body weight, brown and white adipose tissue weights (in hamsters), as well as improving dyslipidemia or obesity [10]. Our results also support this finding of LLA in the active control of behaviors that balance energy intake and expenditure.

In the present study, there was an extending effect in the LLA group on rotarod duration time (Figure 2). After 20 days of treatment, aged mice were able to remain on the rotarod 2.8 times longer than aged controls, while the duration time after 30 days of treatment was prolonged 6.7 times $(p<0.05)$. The rotarod test not only measures effects on muscular endurance and antifatigue but also sensorimotor coordination and flexibility. Thus, the increased ability of aged mice to remain on the rotarod indicates that LLA may play a role in improving fatigueand flexibility. Our data also noted behavioral changes in aged mice administered LLA (Figure 3). LLA treatment resulted in higher spontaneous locomotor activity; the rearing and ambulation behaviors in aged mice were increased 3.4 and 2.2 times, respectively, than in the control mice, suggesting LLA had an excitation effect on the locomotor behavior of mice. Thus, the results from the rotarod and open-field tests provide controlled in vivo evidence of the ergogenic property of LLA. Interestingly, Ganoderma, one of components in LLA, has been reputed to increase youthful vigor and vitality since ancient times. However, it has been reported decreased levels of spontaneous motor activity in a D-galactose mouse-aging model after treatment with Lycium barbarum [11]. These observations suggest that the increased motor activity, as a result of LLA administration, may not simply be produced through the additive effects of the seven components of LLA. Thus the interaction of these components may be worthy of further study.

In the present study, chronic LLA intake caused a significant augmentation of SOD activity in aged mice spleen and an increasing tendency in testes (Figure 4(a)). Reduction in intracellular antioxidant activities, including SOD, GSH, catalase and glutathione-S-transferase, caused by fatigue has been reported in a mouse model $[12,13]$. An increase in mouse spleen and testes SOD following chronic LLA intake may play a role in defense 
against oxidative fatigue stress. Strenuous exercise can result in a dramatic increase in oxygen consumption in the body. Furthermore, it has been reported that strenuous exercise can result in increased reactive oxygen species (ROS) [14-16]. Increased ROS production accelerates muscle fatigue in rat [17], canine [18,19] and mouse [20] models. Thus, chronic administration of LLA may be able to protect aged mice from fatigue-induced oxidative stress.

However, no significant changes in SOD levels in the brain, liver and kidney of aged mice were detected after 4 weeks of LLA supplementation. This indicates that the augmented action of LLA on SOD may be tissue-dependent. In addition, there were no significant changes in serum SOD and GSH levels in aged mice with chronic LLA intake.

On the other hand, chronic supplementation with LLA resulted in a significant GSH activity augmentation in aged mouse liver. GSH is an integral oxidant scavenger, which protects cells and tissues from oxidative damage [21]. It is suggested that increased GSH levels enable detoxification of ROS, which are responsible for exercise-induced protein oxidation, leading to the prevention of fatigue. Thus, LLA administration may improve antioxidant status and delay exercised-induced muscle fatigue. It has also been reported that Crataegus, one of the seven components of LLA, is rich in polyphenols and its extracts inhibit LDL oxidation [22]. Furthermore, it was found that pre-treatment with Crataegus prevented the depletion of reduced $\mathrm{GSH}$ content in the liver of $\mathrm{CCl}_{4}$ injected rats [23], while Ganoderma extract was able to protect the liver from superoxide-induced hepatic damage [24]. Therefore, identification of which component is responsible for the antioxidant property may be needed.

It is suggested that the antifatigue effect of LLA supplementation might be related to antioxidant activity. The antifatigue effect of LLA may be related to the central nervous system, as it potentiates the locomotor activity of the mice. Further study is needed to elucidate the details of the mechanisms involved.

In summary, the present findings suggest that improved ergogenic capacity and alleviated fatigue by LLA supplementation, as demonstrated using the rotarod exercise model, might be due in part to the protective effect against exercise-induced oxidative stress. Thus, LLA most likely functions as an antioxidant agent to improve ergogenic capacity.

\section{Acknowledgements}

We wish to thank International Friendship Trade Co. Ltd., Japan, for donating LLA. We also thank Dr. Pernilla Berin for providing language help.

\section{REFERENCES}

[1] Y. K. Chien, Y. H. Liu and D. G. Massey, "Interleukin-3 and Anti-Aging Medication: A Review," Journal of Hawaii Medicine, Vol. 49, No. 5, 1990, pp. 160-165.

[2] Q. Luo, Y. Cai, J. Yan, M. Sun and H. Corke, "Lycium Barbarum Polysaccharides: Protective Effects against Heat-Induced Damage of Rat Testes and $\mathrm{H}_{2} \mathrm{O}_{2}$ Induced DNA Damage in Mouse Testicular Cell and Beneficial Effect on Sexual Behavior and Reproductive Function of Hemicastrated Rats," Life Science, Vol. 79, No. 7, 2006, pp. 613-621. doi:10.1016/j.lfs.2006.02.012

[3] X. M. Li, Y. L. Ma and X. J. Liu, "Effect of the Lycium Barbarum Polysaccharides on Age-Related Oxidative Stress in Aged Mice," Journal of Ethnopharmacology, Vol. 111, No. 3, 2007, pp. 504-511. doi:10.1016/j.jep.2006.12.024

[4] T. M. Zhu, Q. Chang, L. K. Wong, F. S. Chong and R. C. $\mathrm{Li}$, "Triterpene Antioxidant from Ganoderma lucidum," Phytother Research, Vol. 13, No. 6, 1999, pp. 529-531. doi:10.1002/(SICI)1099-1573(199909)13:6<529::AID-PT R481>3.0.CO;2-X

[5] S. Wachtel-Galor, B. Tomlinson and I. F. Benzie, "Ganoderma lucidum ('Lingzhi'), a Chinese Medicinal Mushroom: Biomarker Responses in a Controlled Human Supplementation Study," The British Journal of Nutrition, Vol. 91, No. 2, 2004, pp. 263-269. doi:10.1079/BJN20041039

[6] P. Scartezzini, F. Antogononi, M. A. Raggi, F. Poli and C. Sabbioni, "Vitamin C Content and Antioxidant Activity of the Fruit and of the Ayurvedic Preparation of Emblica Officinalis Gaertn," Journal of Ethnopharmacology, Vol. 104, No. 1, 2006, pp. 113-118. doi:10.1016/j.jep.2005.08.065

[7] C. C. Kuo, W. Chiang, G. P. Liu, Y. L. Chien, J. Y. Chang, C. K. Lee, S. L. Huang, M. C. Shin and Y. H. Kuo, '2,2'-Diphenyl-1-Picrylhydrazyl Radical Scavenging Active Components from Adlay (Coix Lachryma-Jobi L. var. Ma-Yuen Stapf) Hulls," Journal of Agriculture Food Chemistry, Vol. 50, No. 21, 2002, pp. 5850-5855. doi:10.1021/jf020391w

[8] H. J. Kim and Y. S. Lee, "Identification of New Dicaffeoylquinic Acids from Chrysanthemum Morifolium and Their Antioxidant Activities," Planta Medica, Vol. 71, No. 9, 2005, pp. 871-876. doi:10.1055/s-2005-873115

[9] H. Oku, Y. Ueda and Y. K. Ishiguro, “Antipruritic Effects of the Fruits of Chaenomeles Sinensis," Biological and Pharmaceutical Bulletin, Vol. 26, No. 7, 2003, pp. 10311034. doi:10.1248/bpb.26.1031

[10] D. H. Kuo, C. H. Yeh, P. C. Shieh, K. C. Cheng, F. A. Chen and J. Cheng, "Effect of Shanzha, a Chinese Herbal Product, on Obesity and Dyslipidemia in Hamsters Receiving High-Fat Diet," Journal of Ethnopharmacology, Vol. 124, No. 3, 2009, pp. 544-550. doi:10.1016/j.jep.2009.05.005

[11] H. B. Deng, D. P. Cui, J. M. Jiang, Y. C. Feng, N. S. Cai and D. D. Li, "Inhibiting Effects of Achyranthes Bidentata Polysaccharide and Lycium Barbarum Polysaccharide on Nonenzyme Glycation in D-Galactose Induced Mouse Aging Model," Biomedical and Environmental 
Sciences, Vol. 16, No. 3, 2003, pp. 267-275.

[12] A. Singh, P. S. Naidu, S. Gupta and S. K. Kulkama, "Effect of Natural and Synthetic Antioxidants in a Mouse Model of Chronic Fatigue Syndrome," Journal of Medicinal Food, Vol. 5, No. 4, 2002, pp. 211-220. doi:10.1089/109662002763003366

[13] Y. You, J. Park, H. G. Yoon, Y. H. Lee, K. Hwang, J. Lee, K. Kim, K. W. Lee, S. Shim and W. Jun, "Stimulatory Effects of Ferulic Acid on Endurance Exercise Capacity in Mice," Bioscience, Biotechnology, and Biochemistry, Vol. 73, No. 6, 2009, pp. 1392-1397. doi:10.1271/bbb.90062

[14] K. J. Davies, A. T. Quintanilha, G. A. Brooks and L. Packer, "Free Radicals and Tissue Damage Produced by Exercise," Biochemical and Biophysical Research Communication, Vol. 107, No. 4, 1982, pp. 1198-1205. doi:10.1016/S0006-291X(82)80124-1

[15] M. J. Jackson, R. H. Edwards and M. C. Symons, "Electron Spin Resonance Studies of Intact Mammalian Skeletal Muscle," Biochimica et Biophysica Acta, Vol. 847, No. 2, 1985, pp. 185-190. doi:10.1016/0167-4889(85)90019-9

[16] D. M. Bailey, B. Davies, I. S. Young, M. J. Jackson, G. W. Davison, R. Isaacson and R. S. Richardson, "EPR Spectroscopic Detection of Free Radical Outflow from an Isolated Muscle Bed in Exercising Humans," Journal of Applied Physiology, Vol. 94, No. 5, 2003, pp. 1714-1718.

[17] M. B. Reid, K. E. Haack, K. M. Franchek, P. A. Valberg, L. Kobzik and M. S. West, "Reactive Oxygen in Skeletal Muscle I. Intracellular Oxidant Kinetics and Fatigue in Vitro," Journal of Applied Physiology, Vol. 7, No. 5, 1992, pp. 1797-1804.

[18] E. Nashawati, A. Dimarco and G. Supinski, "Effects Pro- duced by Infusion of a Free Radical-Generating Solution into the Diaphragm," The American Review of Respiratory Disease, Vol. 147, No. 1, 1993, pp. 60-65. doi:10.1164/ajrccm/147.1.60

[19] G. Supinski, D. Nethery, D. Stofan and A. DiMarco, "Effect of Free Radical Scavengers on Diaphragmatic Fatigue," American Journal of Respiratory and Critical Care Medicine, Vol. 155, No. 2, 1997, pp. 622-629.

[20] J. K. Barclay and M. Hansel, "Free Radicals May Contribute to Oxidative Skeletal Muscle Fatigue," Canadian Journal of Physiology and Pharmacology, Vol. 69, No. 2, 1991, pp. 279-284. doi:10.1139/y91-043

[21] T. P. Dalton, Y. Chen, S. N. Schneider, D. W. Nebert and H. G. Shertzer, "Genetically Altered Mice to Evaluate Glutathione Homeostasis in Health and Disease," Free Radical Biology \& Medicine, Vol. 37, No. 10, 2004, pp. 1511-1526. doi:10.1016/j.freeradbiomed.2004.06.040

[22] C. Quettier-Deleu, G. Voiselle, J. C. Fruchart, P. Duriez, E. Teissier, F. Bailleul, J. Vasseur and F. Trotin, "Hawthorn Extracts Inhibit LDL Oxidation," Die Pharmazie, Vol. 58, No. 8, 2003, pp. 577-581.

[23] K. T. Ha, S. J. Yoon, D. Y. Choi, D. W. Kim, J. K. Kim and C. H. Kim, "Protective Effect of Lycium Chinese Fruit on Carbon Tetrachloride-Induced Hepatotoxicity," Journal of Ethnopharmacology, Vol. 96, No. 3, 2005, pp. 529-535. doi:10.1016/j.jep.2004.09.054

[24] Y. H. Shieh, C. F. Liu, Y. K. Huang, J. Y. Yang, I. L. Wu, C. H. Lin and S. C. Li, "Evaluation of the Hepatic and Renal-Protective Effects of Ganoderma lucidum in Mice," The American Journal of Chinese Medicine, Vol. 29, No. 3-4, 2001, pp. 501-507. 